\title{
THEORY OF LOW MASS STARS, BROWN DWARFS AND EXTRA-SOLAR GIANT PLANETS
}

\author{
GILLES CHABRIER AND ISABELLE BARAFFE \\ C.R.A.L., Ecole Normale Supérieure, 69364 Lyon Cedex 07, \\ France \\ chabrier@ens-lyon.fr; ibaraffe@ens-lyon.fr
}

\section{Introduction}

Accurate modeling of the mechanical and thermal properties of very-lowmass stars (VLMS), Brown Dwarfs (BD) and Extra-solar Giant Planets (EGP) is of prior importance for a wide range of physical and astrophysical problems, from the fundamental physics point of view to the astrophysical and cosmological implications. They provide natural laboratories to test the different theories, equations of state, nuclear reaction rates, model atmospheres aimed at describing the physics of dense and cool objects. They represent the largest stellar population in the Galaxy, and thus provide a substantial contribution to the Galactic (disk) mass budget. Finally they represent one of the most intriguing questions in our understanding of the formation of star-like objects: are planet and star formation processes really different ? Is there, and if so what is, a minimum mass for the formation of star-like objects? This field has blossomed recently with the discovery of several brown dwarfs (Nakajima et al. 1995; Rebolo et al., 1995) and numerous exoplanets since 51 Pegasi (Mayor and Queloz 1995; Mayor, this conference), which provide important information to challenge the theory.

\section{Theoretical improvements}

VLMS, or M-dwarfs, defined hereafter as objects with masses below $0.6 M_{\odot}$, are compact objects, with characteristic radii in the range $\sim 0.1-0.6 R_{\odot}$. Their central densities and temperatures are respectively of the order of $\rho_{c} \approx 10-10^{3} \mathrm{gcm}^{-3}$ and $T_{c} \approx 10^{6}-10^{7} \mathrm{~K}$, so that the stellar interior represents a strongly correlated plasma. BD's are defined as objects not

T.R. Bedding et al. (eds.),

Fundamental Stellar Properties: The Interaction between Observation and Theory, 331-340.

(C) 1997 IAU. Printed in the Netherlands. 
massive enough for their central temperature to sustain hydrogen burning. This characteristic stems from the onset of degeneracy in the contracting protostar, which prevents an increase of the temperature as contraction proceeds. A BD therefore never reaches thermal equilibrium and cools down for its whole life. The hydrogen-burning minimum mass (HBMM) is $\sim 0.07-0.09 M_{\odot}$, depending on the initial composition from $\mathrm{Z}=\mathrm{Z}_{\odot}$ to $\mathrm{Z}=0$, respectively (Chabrier \& Baraffe, 1997). The minimum mass depends on stellar formation theory and is still uncertain (Hubbard, 1994). Brown dwarfs bridge the gap in the observed mass-distribution of astrophysical objects between the lowest-mass star and the largest solar planet, Jupiter $\left(M_{J}=0.001 M_{\odot}\right)$. The distinction between $\mathrm{BD}$ and Giant Planets is based on different formation scenarios, but their inner structure (apart from the central rocky core in planets) and spectral signatures are both governed by the same physics.

The effective temperatures of these low-mass objects are below $T_{\text {eff }} \approx$ $5000 \mathrm{~K}$, down to $\sim 100 \mathrm{~K}$ for BDs and EGPs, and surface gravities $g=$ $G M_{\star} / R_{\star}^{2}$ are in the range $\log g \approx 3.5-5.5$. The low effective temperature allows the presence of stable molecules in the atmosphere (see Baraffe \& Allard, these proceedings). The presence of these bands complicates tremendously the treatment of radiative transfer, not only because of the numerous transitions to include in the calculations, but also because the molecular absorption coefficients strongly depend on the frequency. Moreover molecular recombination in the interior $\left(2 \mathrm{H} \rightarrow \mathrm{H}_{2}\right)$ leads to a decrease of the adiabatic gradient so that convection penetrates deeply into the optically-thin atmospheric layers. Because of these physical processes, the grey-approximation is no longer valid below $T_{\text {eff }} \sim 5000 \mathrm{~K}$, as shown by several authors (Allard, 1995; Saumon et al., 1994; Chabrier et al., 1996; Chabrier \& Baraffe, 1997).

These conditions show that the modeling of low-mass objects requires a correct description of non-ideal effects in the interior, as well for the equation of state (EOS) as for the screening factors of the nuclear rates, a derivation of accurate models for dense and cool atmospheres, where molecular opacity becomes eventually the main source of absorption, and consistent (non-grey) boundary conditions between the atmosphere and the interior along evolution.

A new EOS, devoted to the description of low-mass stars and giant planets, has been derived recently, which presents a consistent treatment of pressure ionization (Saumon, Chabrier \& VanHorn 1995; SCVH), altough based on the so-called ideal-volume mixing between hydrogen and helium (see SCVH). Improvement in the field of cool atmosphere models has blossomed within the past few years with the work of Allard and Hauschildt $(1995 ; 1997)$, Brett $(1995)$, and Saumon et al. (1994). Non-grey atmosphere 
models now extend down to $T_{\text {eff }}=900 \mathrm{~K}$, since the discovery of the first cool BD GL 299B offered a stringent test for such models (Allard et al. 1996; Tsuji et al. 1996a; Marley et al., 1996).

A detailed description of the recent improvement in the theory of lowmass objects (LMS and BDs), and a comparison with standard grey-like treatments, is given in a recent paper by Chabrier \& Baraffe (1997). The modelization of VLMS, BDs and EGPs and the confrontation with observations is examined below.

\subsection{VLM STARS}

In spite of considerable progress in stellar theory - internal structure, model atmospheres and evolution - all the VLMS models so far failed to reproduce accurately the observed color-magnitude diagrams (CMD) of disk or halo stars below $\sim 4000 \mathrm{~K}$, i.e. $\sim 0.4-0.6 M_{\odot}$, depending on the metallicity. All the models predicted too hot an effective temperature for a given luminosity, i.e. were too blue compared to the observations by at least one magnitude. Such a disagreement stemmed essentially from shortcomings both in the physics of the interior, i.e. equation of state (EOS) and thus massradius relationship and adiabatic gradient, and in the atmosphere, since all models were based on grey atmospheres and approximate outer boundary conditions. Important progress has been made recently in this field with the derivation of evolutionary models based on a consistent treatment between the interior and the atmosphere profile (Baraffe, Chabrier, Allard \& Hauschildt 1995, 1997; Chabrier, Baraffe \& Plez 1996). The effect of the outer boundary condition on the mass- $T_{\text {eff }}$ relationship has been examined in detail by Chabrier \& Baraffe (1997). These authors have made comparison with models based on different grey-like treatments and have shown convincingly that such treatments (which imply a $T(\tau)$ relationship as an external boundary condition) are incorrect, or at best highly unreliable as soon as molecular formation sets in, i.e. for any object below $T_{\text {eff }} \lesssim 5000$ K, i.e. $m \sim 0.5 M_{\odot}$.

The LMS models based on the afore-mentioned updated physics and consistent (non-grey) boundary condition now reach quantitative agreement with observations for both the disk and the halo stellar population down to the bottom of the main sequence (Figure 1). For the disk population the models reproduce the observed color-magnitude diagrams both in the infrared $\left(M_{K}\right.$ vs $\left.(\mathrm{I}-\mathrm{K})\right)$ and in the optical $\left(M_{V}\right.$ vs $(\mathrm{V}-\mathrm{I})$ ) (Baraffe et al., $1997 \mathrm{~b})$, although below $0.1 M_{\odot}, M_{V} \gtrsim 15$, the models are still too blue by $\sim 0.2-0.4 \mathrm{mag}$ in $(\mathrm{V}-\mathrm{I})$, as illustrated in Figure 1 . This is likely to stem from the still inaccurate $\mathrm{TiO}$ line list which shapes the flux in the optical, or possibly from the onset of grain formation for solar metallicity (see 


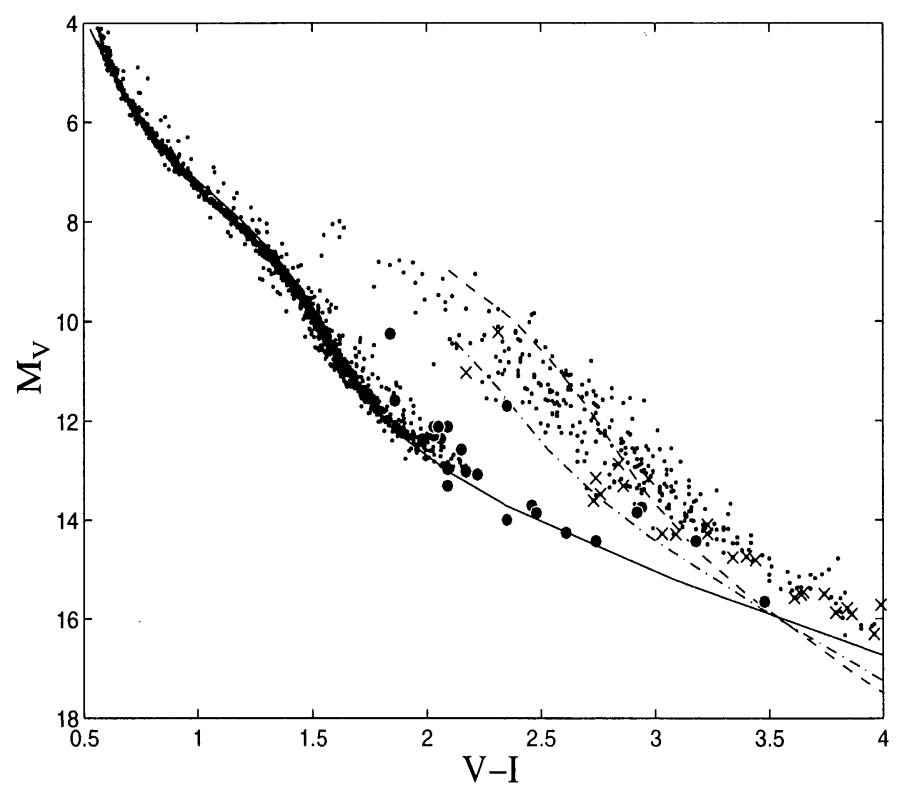

Figure 1. $\mathrm{M}_{V}-(\mathrm{V}-\mathrm{I})$ diagram for different metallicities : $[\mathrm{M} / \mathrm{H}]=-1.5$ (solid line), $[\mathrm{M} / \mathrm{H}]=-0.5$ (dash-dot) and $[\mathrm{M} / \mathrm{H}]=0$ (dash). The Main Sequence of the Globular Cluster NGC6397 from Cool et al. (1996) is shown on the left part. Subdwarf halo field stars from Monet et al. (1992) are indicated by full circles, as well as disk M-dwarfs of Monet et al. (1992) (crosses) and Dahn et al. (1995) (dots on the right hand side)

Baraffe \& Allard, these proceedings). More importantly, the models are in excellent agreement with the observationally-determined mass-magnitude relationship (Henry \& McCarthy, 1993) both in the infrared and in the optical (Chabrier, Baraffe \& Plez, 1996; Baraffe et al., 1997b) as shown in the present Figure 2 and in Figure 1 of Baraffe \& Allard (these proceedings). For metal-depleted populations, the models are in remarkable agreement with the main sequences of globular clusters observed with the HST nearly down to the bottom of the main sequence and with the halo field subdwarf sequence, as shown in Figure 1 (Baraffe et al., 1997a). We stress that, since LMS are essentially fully convective below $\sim 0.4 M_{\odot}$ (Chabrier \& Baraffe, 1997), the models are not hampered by any adjustable parameter and the agreement between theory and observation reflects directly the reliability of the physics entering the models. These models yield the derivation of reliable mass-functions for the disk population (Méra, Chabrier \& Baraffe, 1996) and for globular clusters and halo field stars down to the brown dwarf limit (Chabrier and Méra, 1997). 


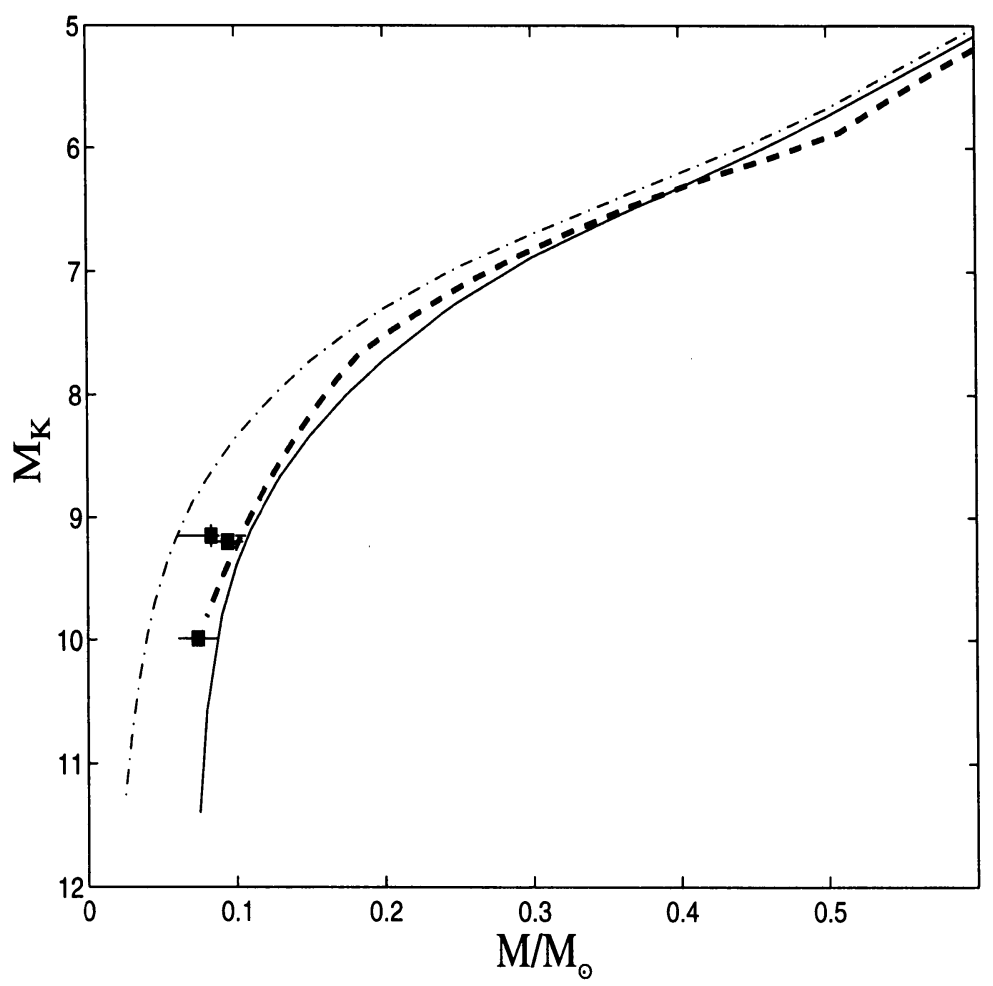

Figure 2. Mass-luminosity relationship in the $K$-band. The dash-line is the fit of the observationally-determined relation (Henry \& McCarthy, 1993; HMc93), the solid line is based on the most recent models for solar metallicity (Baraffe et al., 1997b) for $t=5$ Gyr, whereas the dash-dot line is the same models for $t=0.1$ Gyr (the ZAMS age for $0.1 M_{\odot}$ is about $0.5 \mathrm{Gyr}$ ). Note that the three lowest-mass objects observed by HMc93, shown on the figure with their error bars, all exhibit strong surface $\left(\mathrm{H}_{\alpha}\right.$-emission $)$ and coronal ( $X$-emission) activity. Although this is not a proof of a young age, it is a strong hint and is supported by the present analysis.

\subsection{BROWN DWARFS}

The recent discovery of substellar objects enables us to test the reliability of the theory in the BD regime. The first young brown dwarfs were identified in the Pleiades star cluster (Rebolo et al. 1995), namely Teide 1 and Calar 3 , which both have retained their initial Lithium abundance (Rebolo et al., 1996). Their extremely low luminosity $\left(\log L / L_{\odot} \sim-3.1\right)$ combined with the presence of lithium yields masses $m \sim 0.05 M_{\odot}$, well below the hydrogenburning minimum mass (Rebolo et al., 1996; Baraffe et al, 1997b). Given the large effective temperature of these two BDs $\left(T_{\text {eff }} \sim 2500 \mathrm{~K}\right)$, due to their young age $\left(\sim 10^{8} \mathrm{yrs}\right)$, the most stringent test for extremely cool models is provided by Gl 229B (Nakajima et al. 1996). The presence of methane in its 
infrared spectrum clearly reveals its substellar nature, since $\mathrm{CH}_{4}$ absorption appears below $1600 \mathrm{~K}$ (Tsuji et al., 1996a) and a star at the hydrogen burning limit has an effective temperature of about $2000 \mathrm{~K}$ (Baraffe et al. 1995). Comparison of synthetic and observed spectra yields an effective temperature of $\sim 1000 K$ (Allard et al. 1996; Tsuji et al. 1996a; Marley et al. 1996). Uncertainties in the age of the system and in the temperature of Gl229B yield some indetermination for the mass. Evolutionary calculations based on the afore-mentioned synthetic spectra and non-grey atmosphere models yield the most likely solution $M \approx 0.04-0.055 M_{\odot}$ for an age $\sim 5$ Gyr, similar to our solar system (Allard et al. 1996; Marley et al., 1996).

Figure 3 presents different isochrones as a function of metallicity (Baraffe et al. 1997b) at the bottom and below the main sequence in IR colors. The K-limit magnitudes corresponding to the HBMM are indicated by full circles. The blue loop displayed in the substellar domain stems from collisioninduced absorption of $\mathrm{H}_{2}$, and $\mathrm{CH}_{4}$ absorption for solar-metallicity, in the K-band (Saumon et al., 1994; Baraffe et al., 1997a,b) which shifts the flux back to shorter wavelengths. We predict this blueshift in IR-colors, whereas optical colors keep reddening almost linearly, to be the most important photometric signature of the transition from the stellar to the sub-stellar domain. We have made predictions of such signatures in the NICMOS filters, which should be verifiable in a very-near future (Baraffe et al., 1997a).

\subsection{EXTRASOLAR GIANT PLANETS}

A general theory of EGPs, from 0.3 to 15 Jupiter masses, and the possibility of detection by existing and future observational projects, has been performed recently by Saumon et al. (1996). These calculations take root in the theory of solar giant planets derived previously (Chabrier et al., 1992; Guillot et al. 1995). In this first generation of calculations, the spectral emission of the EGP and of the parent star is approximated by a black body distribution. Although weakened by this approximation, the predictions made in Saumon et al. (1996) represent the first benchmark in search strategies for the detection of EGPs. They have been applied more specifically to the case of $51 \mathrm{PegA}$ to demonstrate the stability of giant planets orbiting nearby stars (Guillot et al., 1996).

More recent calculations by Marley et al. and Allard et al. do now predict synthetic spectra in the substellar domain from $T_{\text {eff }} \sim 2000 \mathrm{~K}$ down to $\sim 300 \mathrm{~K}$. The predicted absolute fluxes of BD or EGP (Allard et al. 1997) are displayed in Fig. 3 of Baraffe \& Allard (these proceedings) and compared to the sensitivity of ground and space-based observing platforms.

An extra-degree of complication in the atmosphere of objects below $T_{e f f} \sim 2600 \mathrm{~K}$ is due to the onset of grain formation, as suggested by Tsuji 


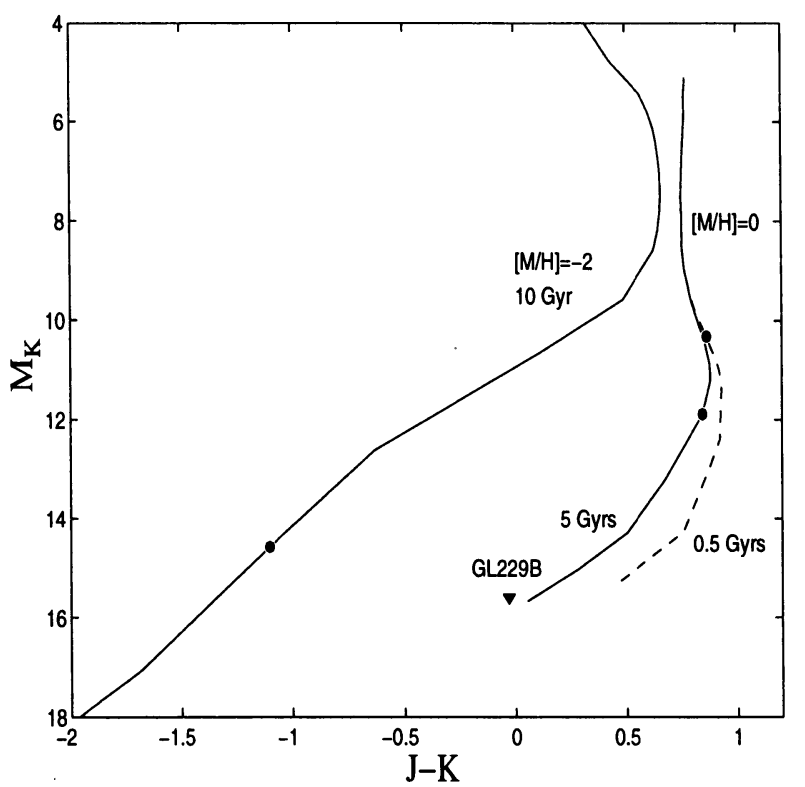

Figure 3. Color-magnitude diagram in the near IR for the metallicity and ages indicated on the figure. The full circles on the curve indicate the stellar/sub-stellar transition.

and collaborators (Tsuji et al. 1996b). This is illustrated in Figure 4 which displays the effective temperature and the radius of various exoplanets discovered recently with the domain of condensation (bars on the right hand side) of various compounds (Burrows et al., 1997).

\section{Conclusion}

The recent improvements in the description of the mechanical and thermal properties of cool, compact objects, and of their photometric signature now provide solid grounds to analyse the observations and make reliable predictions. The three essential inputs in the theory of these objects are an accurate EOS, with a reliable treatment of non-ideal effects and pressure ionization, synthetic spectra with accurate molecular absorption coefficients in the optical and the IR, and consistent evolutionary calculations with correct (non-grey) boundary conditions between the atmosphere and the interior profiles.

This improved theory of LMS and substellar objects allows now the derivation of reliable stellar mass-functions down to the brown dwarf limit and, depending on the rate of discovery of substellar objects in the near future, will yield eventually the brown dwarf mass function, in connection with microlensing observations. This will bring new insight on the still 

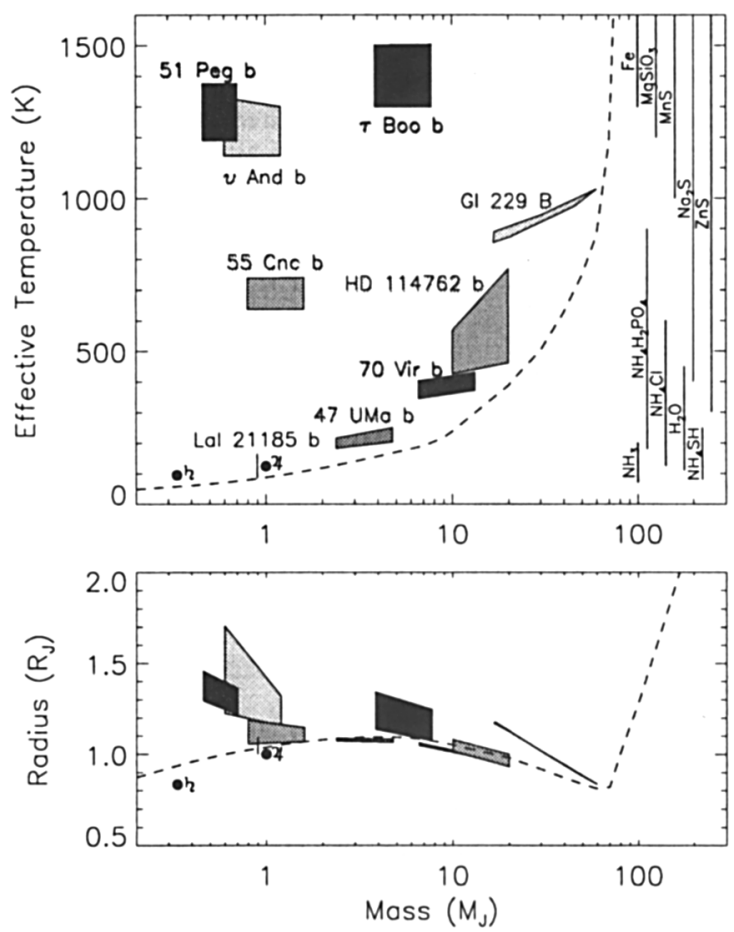

Figure 4. $T_{\text {eff }}$ and radius as a function of mass for several EGPs (kindly provided by A. Burrows). Jupiter and Saturn are also indicated by their usual symbols. The bars on the right hand side show the domain of condensation of different species.

unsolved problem of stellar versus planetary formation.

The derivation of cool atmosphere models and synthetic spectra including grain formation represents the next substantial improvement. Work is already under progress in this field, both in the Tucson group and in the Lyon-Wichita group, and promises more exciting results in the near future.

\section{References}

Allard, F., 1995, The bottom of the main-sequence and below, Ed. C. Tinney, Springer Verlag

Allard, F., and Hauschildt, P. H., 1995, ApJ, 445, 433

Allard, F., Hauschildt, P.H., Baraffe, I., Chabrier, G., 1996, ApJ, 465, L123

Allard, F., Hauschildt, P.H., Alexander D. R., Starrfield, S., 1997, ARA\&A, 35, 137

Baraffe, I., Chabrier, G., Allard, F., Hauschildt P., 1995, ApJ, 446, L35

Baraffe, I., Chabrier, G., Allard, F., Hauschildt P., 1997a, A\&A, in press

Baraffe, I., Chabrier, G., Allard, F., Hauschildt P., 1997b, in preparation

Brett, J.M., 1995, A\&A, 295, 736

Burrows A. et al., 1997, in preparation 
Chabrier, G., Saumon, D., Hubbard, W.B. \& and Lunine, J.I., 1992, ApJ, 391, 317

Chabrier, G., Baraffe, I., Plez, B., 1996, ApJ, 459, L91

Chabrier, G., Baraffe, I., 1997, A\&A, in press

Chabrier, G. \& Méra, D., 1997, A\&A, in press

Cool, A. M., Piotto, G., King, I.R., 1996, ApJ, 468, 655

Dahn, C.C., Liebert, J., Harris, H.C., Guetter, H.H., 1995, The bottom of the mainsequence and below, Ed. C. Tinney

Guillot, T., Chabrier, G., Gautier, D., Morel P., 1995, ApJ, 450, 463

Guillot, T., Burrows, A., Lunine, J.I., Saumon, D. 1996, ApJ, 459, L35

Henry, T.D., and McCarthy, D.W.Jr, 1993, AJ, 106, 773

Hubbard. W. B., 1994: the Equation of State in Astrophysics, Ed. G. Chabrier and E. Schatzman, Cambridge University Press

Marley et al., 1996, Science, 272, 1919

Mayor, M., Queloz, D., 1995, Nature, 378, 355

Méra, D., Chabrier, G. and I. Baraffe, 1996, ApJ, 459, L87

Monet D.G. et al., 1992, Astron. J., 103, 638

Nakajima et al., 1995, Nature, 378, 463

Rebolo, R., Zapatero Osorio, M.R., Martin, E.L., 1995, Nature, 377, 129

Rebolo, R., Martin, E.L., Basri, G., Marcy, G.W. \& Zapatero Osorio, M.R., 1996, ApJ, 469, L53

Saumon, D., Bergeron, P., Lunine, L.I., Hubbard, W.B., Burrows, A., 1994, ApJ, 424, 333

Saumon, D., Hubbard, W.B., Burrows, A., Lunine, J.I. and Chabrier, G., ApJ, 1996, 460, 993

Saumon, D., Chabrier, G., VanHorn, H.M., 1995, ApJS, 99, 713

Tsuji, T., Ohnaka, K., Aoki, W., Nakajima. T., 1996a, A\&A, 308, L29

Tsuji, T., Ohnaka, K., Aoki, W., 1996b, A\&A, 305, L1

\section{DISCUSSION}

PIERRE MAXTED: You suggested that two of the brown dwarfs seen in the Pleiades are binary stars since they sit so far above the isochrones in the HR diagram. Is there any independent observational evidence of binarity in these stars?

GILLES CHABRIER: The groups of Rafael Rebolo and Gibor Basri, who suggested the possible binarity, are examinating this possibility but, as far as I know, they don't have a definitive answer yet.

JゆRGEN CHRISTENSEN-DALSGAARD: Would it be fair to ask what the MACHO objects are, if not brown dwarfs?

GILLES CHABRIER: As we've shown in a recent paper (ApJ 468 L21), they can be explained by halo white dwarfs, providing some stringent conditions are met. I will illustrate this point in my white dwarf talk.

FLAVIO FUSI PECCI: You have shown nice fits of observed faint main sequences with Galactic globulars and claim that the quality of the fits is much better than obtained so far, thanks to the improved quality of your models compared to previous ones. I have seen similar fits obtained, for instance, for NGC 6397 (HST Data) by Alexander et al. (1997 A\&A, 31790 ) using their own models - could you schematically explain which are the main differences between 
the two sets and how you determine and evaluate the quality of the best fitting solution?

GILLES CHABRIER: A detailed answer is given in our papers (Chabrier \& Baraffe 1997; Baraffe et al. 1997a). To summarize, there are five important differences:

1. Our models rely on consistent non-grey boundary conditions, whereas the Teramo models use a grey condition, based on $\mathrm{T}(\tau)$ approximation. Even when modified (or adjusted), the grey approximation is basically not correct/reliable for LMS, because of the very physics characteristic of these objects (see the present review).

2. With the same physics, and no adjustable parameter, our models reproduce all the observed sequences from $\mathrm{Fe} / \mathrm{H}=-2.2$ (M 15) to -1.0 ( $\omega$ Cen) and even -0.5 (47 Tuc), not just NGC 6397.

3. The Teramo models assume $[M / H]=[\mathrm{Fe} / \mathrm{H}]$ which is not correct for metal-depleted stars, because of the $O / F e$ enrichment, which yields $[M / H]=[O / H]$.

4. The Teramo models rely on previous (so-called "Base") colors by Allard \& Hauschildt which overestimate the opacity (because of the Straight mean approximation, see Allard et al., 1997, ARA\&A) and are now abandoned. Moreover the bolometric corrections have been shifted arbitrarily to recover the Kurucz values at high temperatures (see their $\$ 4$ ).

5. Our models use consistent reddening corrections, calculated from Allard's synthetic spectra. These corrections $(E(V-I)=0.22)$ are in excellent agreement with the ones used by the observers $(E(V-I)=0.23$; Cool et al., 1996). The ones used by the Teramo group $(E(V-I)=0.19$, see their Fig. 7) differ significantly. With the reddening and metallicity quoted by the observers, their models are too red w.r.t. the observations (see e.g. Fig 5 of Baraffe et al., 1997a). 